\title{
Prenatal Exposure to Elevated NT3 Disrupts Synaptic Selectivity in the Spinal Cord
}

\author{
Zhi Wang, ${ }^{1}$ Ling Ying Li, ${ }^{1}$ Michael D. Taylor, ${ }^{2}$ Douglas E. Wright, ${ }^{2}$ and Eric Frank ${ }^{1}$ \\ ${ }^{1}$ Department of Physiology, Tufts University School of Medicine, Boston, Massachusetts 02111, and 2Department of Anatomy and Cell Biology, University \\ of Kansas Medical Center, Kansas City, Kansas 66160
}

\begin{abstract}
Monosynaptic connections between muscle spindle (Ia) afferents and motoneurons (MNs), the central portion of the stretch reflex circuit, are highly specific, but the mechanisms underlying this specificity are primarily unknown. In this study, we report that embryonic overexpression of neurotrophin-3 (NT3) in muscles disrupts the development of these specific Ia-MN connections, using transgenic (mlc/NT3) mice that express elevated levels of NT3 in muscles during development. In mlc/NT3 mice, there is a substantial increase in the amplitudes of monosynaptic EPSPs evoked by Ia afferents in MNs as measured with extracellular recordings from ventral roots. Despite this increased functional projection of Ia afferents, there is no obvious change in the anatomical density of Ia projections into the ventral horn of the spinal cord. Intracellular recordings from MNs revealed a major disruption in the pattern of Ia-MN connections. In addition to the normal connections between Ia afferents and MNs supplying the same muscle, there were also strong monosynaptic inputs from Ia afferents supplying unrelated muscles, which explains the increase seen in extracellular recordings. There was also a large variability in the strength of Ia input to individual MNs, both from correct and incorrect Ia afferents. Postnatal muscular administration of NT3 did not cause these changes in connectivity. These results indicate that prenatal exposure to elevated levels of NT3 disrupts the normal mechanisms responsible for synaptic selectivity in the stretch reflex circuit.
\end{abstract}

Key words: synaptic specificity; stretch reflex; neurotrophin-3; Ia fibers; spinal cord; EPSP

\section{Introduction}

For the nervous system to function correctly, specific patterns of synaptic connections must be established between appropriate groups of neurons. One well characterized example is the monosynaptic connections between muscle spindle sensory afferents (Ia fibers) and spinal motoneurons (MNs), the central pathway of the neuronal circuit mediating the simple stretch reflex. In this reflex, Ia afferents innervating one muscle make strong monosynaptic excitatory synapses with MNs supplying the same muscle (homonymous connections) but make few, if any, monosynaptic connections with MNs supplying antagonist or functionally unrelated muscles (Eccles et al., 1957; Frank and Westerfield, 1983; Lichtman et al., 1984). Molecular cues within the target tissue are likely to be critical in specifying the pattern of these connections (Chen et al., 2003), but to date, the identity of these molecules is unknown. The experiments reported here show that elevated levels of neurotrophin-3 (NT3) during development disrupt synaptic specificity in this system and may therefore be useful in exploring the mechanisms responsible for this specificity.

NT3 is a member of the nerve growth factor-related family of

Received Jan. 17, 2007; revised Feb. 13, 2007; accepted March 5, 2007.

This work was supported by a grant from the National Institutes of Health. We thank Danielle Gelfand and Chaney Stewman for the breeding and genotyping of animals, Bethany Kiernan for help with the histology, and David Harmen and Nicholas Priebe for help with the statistical evaluation of EPSP amplitude histograms.

Correspondence should be addressed to Dr. Eric Frank, Department of Physiology, Tufts University School of Medicine, 136 Harrison Avenue, Boston, MA 02111. E-mail: eric.frank@tufts.edu. D01:10.1523/JNEUROSCI.0197-07.2007

Copyright $\odot 2007$ Society for Neuroscience $\quad$ 0270-6474/07/273686-09\$15.00/0 neurotrophins that regulate neuronal survival, differentiation, and axonal growth (Hory-Lee et al., 1993; Lindsay, 1996; Coppola et al., 2001; Zhou et al., 2003). For proprioceptive sensory neurons supplying muscles, NT3 is best known for its key role in determining the number of these neurons that survive the period of naturally occurring cell death. Deletion of the genes coding for NT3 or its specific receptor trkC in mice leads to a virtually complete loss of these sensory neurons (Ernfors et al., 1994; Klein, 1994; Tessarollo et al., 1994). Injection of NT3 antibodies into peripheral tissues reduces the number of proprioceptive neurons (Oakley et al., 1995), suggesting that target-derived NT3, which is produced by skeletal muscle fibers, is critical for this survival function. Elevated NT3 in muscles during prenatal development increases the number of surviving Ia afferents, demonstrating that normal levels are limiting (Oakley et al., 1997; Wright et al., 1997). In addition to its effects on neuronal survival, NT3 stimulates branching of proprioceptive axons (Lentz et al., 1999; Ulupinar et al., 2000; Ozdinler et al., 2004) and is critical for the expression of the ETS transcription factor Er81, which promotes the growth of Ia axon collaterals into the ventral horn (Arber et al., 2000; Patel et al., 2003). Together, these studies indicate that NT3 plays several roles throughout the development of sensory and motor neurons in the spinal cord.

In examining the influence of NT3 on the formation of sensory motor synapses in mice lacking the transcription factor Er81 (Li et al., 2006), we discovered an additional unexpected role of this neurotrophin in the development of the stretch reflex circuit. Prenatal exposure to elevated levels of NT3 results in a major 
disruption of the pattern of monosynaptic connections between Ia afferents and MNs. Further analysis of synaptic potential amplitudes suggests that elevated NT3 levels influence the susceptibility of MNs to receive Ia input, thereby disrupting the ability of $\mathrm{MNs}$ to discriminate between correct and incorrect Ia inputs.

\section{Materials and Methods}

Animals. Transgenic mlc/NT3 mice were generated using a construct with the NT3 gene driven by a portion of the myosin light chain-1 (mlc) promoter. The mlc promoter is expressed exclusively in skeletal muscle beginning at about embryonic day 14 (E14). All mlc/NT3 mice used were derived from the founder line 7310 (Taylor et al., 2001) and were bred with C57BL/6 mice. For all mice, postnatal day 0 (P0) was defined as the first $24 \mathrm{~h}$ period after birth. All mice used in these studies were P7-P9 neonates. Each mouse was genotyped using the PCR procedure (Taylor et al., 2001). The following primers were used to identify the $\mathrm{mlc} / \mathrm{NT3}$ transgene: NT3 reverse primer, 5' -TTGATCCATGCTGTTGCCTT-3'; MLC1 forward primer, $5^{\prime}$-ACTGTACTCCCTAGAAAG-3' . All research was approved by the Institutional Animal Care and Use Committee at Tufts University School of Medicine and conformed to National Institutes of Health (NIH) guidelines. Experimenters were blinded to the genotypes of the mice during all recording and subsequent electrophysiological and morphological analyses.

Preparation. An isolated spinal cord preparation was used for all experiments. Details of this preparation have been described previously (Mears and Frank, 1997). Briefly, neonatal mice were anesthetized with hypothermia, perfused with cold saline, decapitated, skinned, and eviscerated. Dissection was performed in recirculating oxygenated $\left(95 \% \mathrm{O}_{2}\right.$, $\left.5 \% \mathrm{CO}_{2}\right)$ saline at room temperature $\left(22-25^{\circ} \mathrm{C}\right)$. The solution used for dissection and recording contained the following (in $\mathrm{mm}$ ): $127 \mathrm{NaCl}, 1.9$ $\mathrm{KCl}, 1.2 \mathrm{KH}_{2} \mathrm{PO}_{4}, 2 \mathrm{CaCl}_{2}, 1 \mathrm{MgSO}_{4}, 25 \mathrm{NaHCO}_{3}$, and 20.5 dextrose. The hindlimb nerves including quadriceps, adductors, ankle plantar flexors (APF; soleus and medial and lateral heads of the gastrocnemius muscle), and flexor digitorum longus (FDL) were dissected in continuity with the spinal cord. The isolated cord was hemisected along the dorsoventral midline and was positioned in the recording chamber with its cut medial surface uppermost.

Electrophysiology. For extracellular recordings, synaptic potentials elicited by stimulation of muscle nerves were recorded from lumbosacral ventral roots L3-L5 with a tight-fitting glass suction electrode in close proximity to the cord. $\mathrm{MgSO}_{4}$ was increased to $2 \mathrm{~mm}$ to reduce the generation of orthodromic action potentials in MNs (see Fig. 1). For intracellular recordings, MNs were impaled with glass micropipettes (100-180 M $\Omega$ ) filled with $2 \mathrm{M}$ potassium methylsulfate, $500 \mathrm{~mm}$ lidocaine $N$-ethyl bromide (QX-314; to block antidromic action potentials; Alomon Labs, Jerusalem, Israel), and $0.5 \%$ fast green (to facilitate placement of the micropipette). MNs were identified by antidromic action potentials evoked by stimulation of the appropriate muscle nerve. QX-314, diffusing from the electrode tip, blocked somatic action potentials after several minutes, facilitating the measurement of homonymous synaptic inputs. A small residual antidromic potential remained in some of the homonymous recordings (Fig. 3). Measurements from only those MNs with a resting potential more negative than $-50 \mathrm{mV}$ and in which synaptic potential amplitudes were stable for at least several minutes are included here.

Muscle nerves were stimulated via suction electrodes with square pulses of $0.1 \mathrm{~ms}$ duration at supramaximal levels $(<7 \mathrm{~V})$. The stimulation frequency was typically $0.33 \mathrm{~Hz}$, which produced little or no synaptic fatigue. The resulting potentials, filtered at $3-10 \mathrm{kHz}$, were recorded digitally at $10-20 \mathrm{kHz}$. Individual traces (5-50 traces, depending on the variability of the responses) were averaged either on- or off-line and stored for subsequent analysis using custom software (LabVIEW; National Instruments, Austin, TX).

To measure the monosynaptic component of each synaptic potential, we scaled a superimposed model trace (recorded in response to stimulation of the same muscle nerve but at just suprathreshold strength) to fit the rising phase of the potential to be measured (Sah and Frank, 1984; Mendelson and Frank, 1991; Arber et al., 2000). Fluctuations in synaptic latency for an individual response (see Fig. 5) were calculated by measuring the latency of $>30$ single traces. A least-squares linear fit was performed of the baseline (just before the synaptic potential began) and the approximately linear portion of the rising phase of each trace; the latency for that trace was then taken as the intersection of these two straight lines.

The statistical validity of the difference in the distribution of synaptic potential amplitudes (see Fig. 8) was calculated using the bootstrap method (Sokal and Rohlf, 1995). The data sets in each histogram were randomly sampled with replacement 10,000 times. The SD of the mean and the number of data points below a given threshold were calculated for each of the 10,000 trials. The degree of overlap between the distributions of SDs (or number of points below threshold) of wild-type and mlc/NT3 mice then provided a measure of the probability that these parameters were significantly different from one another.

Labeling of central projections of sensory neurons in the spinal cord. Central projections of sensory neurons in the spinal cord were labeled with fluorescent dextran. The central end of the L3 dorsal root was drawn into a tight-fitting glass capillary that was then backfilled with an aqueous solution of $100 \mathrm{mg} / \mathrm{ml}, 3000$ molecular weight lysine-fixable fluorescein dextran (Invitrogen, Eugene, OR) with 1\% lysolecithin (Sigma, St. Louis, $\mathrm{MO}$ ). Labeling was allowed to proceed overnight at $25^{\circ} \mathrm{C}$, followed by overnight fixation in $4 \%$ paraformaldehyde in $0.1 \mathrm{~m}$ phosphate buffer at $4^{\circ} \mathrm{C}$. Serial transverse sections of the spinal cord were cut at $50 \mu \mathrm{m}$ on a cryostat, and images were captured using a confocal microscope (TCS SP2; Leica, Nussloch, Germany).

To assess the density of these central projections in the ventral horn, we chose a representative section from each cord at the rostrocaudal position with greatest sensory fiber labeling in the ventral horn. A stack of 16 optical sections was made and combined digitally with the confocal microscope, and the combined image was analyzed using Image (NIH, Bethesda, MD). An average background intensity was determined from an unlabeled area of the lateral gray matter (see Fig. 2, dotted lines), and this intensity was used as a threshold for counting labeled pixels. A region of interest (ROI) was drawn enclosing the area of Ia axon arborization in the ventral horn (see Fig. 2, dashed lines), and the percentage of pixels above threshold within the ROI was calculated.

NT3 protein determination. NT3 protein in quadriceps muscles was detected using an ELISA for NT3 (NT3 $\mathrm{E}_{\text {max }}$ Immunoassay System; Promega, Madison, WI). Briefly, the quadriceps muscles from P6-P8 mice were quickly excised and frozen in liquid nitrogen. Tissue samples were extracted with Tissue-PE LBTM (Geno Technology, St. Louis, MO) with Complete Mini Protease inhibitor cocktail tablets (Roche Diagnostics, Mannheim, Germany) and subjected to protein assay using the Total Protein kit, Micro Lowey TP0300 (Sigma-Aldrich, St. Louis, MO). Equal amounts of protein extracts $(10 \mu \mathrm{g})$ were analyzed in duplicate according to the manufacturer's instructions. Test samples were compared with the linear part of a standard NT3 curve to determine the NT3 concentration in picograms per $100 \mu \mathrm{g}$ of extracted protein.

NT3 administration. Recombinant NT3 (a gift from Regeneron Pharmaceuticals, Tarrytown, NY) diluted in PBS was injected $(5 \mu \mathrm{g} / \mathrm{ml})$ in a volume of 1-4 $\mu \mathrm{l}$ (depending on the ages of the mice) into the right proximal hindlimb of wild-type mice on P1, P3, P5, and P7.

\section{Results}

Phenotypic behavior with elevated muscular NT3 in mlc/NT3 transgenic mice

A distinctive behavioral phenotype of mlc/NT3 mice is that by P5, these mice clasp their hindlimbs together when they are lifted by their tails (Taylor et al., 2001). This "clasping phenotype" provided a convenient check on the genotype of each animal, confirming the genotyping based on PCR. We also confirmed that these mice have elevated levels of NT3 in their hindlimb muscles (Taylor et al., 2001). Analysis of NT3 in extracts of these muscles by ELISA indicated that in P6-P8 neonates, the NT3 level was more than four times larger than normal wild-type littermates (mlc/NT3: $3.10 \pm 0.31 \mathrm{pg} / \mu \mathrm{g}$ protein, $n=4$; wild type: $0.69 \pm$ $0.12 \mathrm{pg} / \mu \mathrm{g}$ protein, $n=5 ; p<0.001)$. A primary cause of the 


\section{Wild-Type}
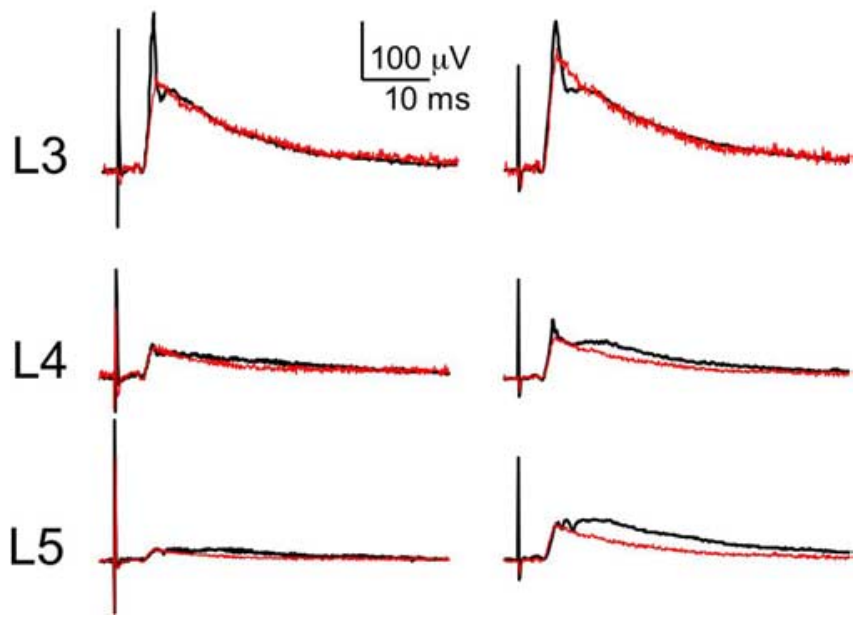

Figure 1. Representative excitatory EPSPs (black traces) recorded extracellularly from lumbar spinal cord ventral roots $L 3-L 5$ in response to stimulation of the $Q$ or Add muscle nerve in wild-type and $\mathrm{ml} / \mathrm{NT} 3$ mice at $\mathrm{P} 7$. Ventral root recordings monitor the average response in all MNs projecting in the root. At $L 3$ and $L 4$, orthodromic action potentials were evoked in some MNs, obscuring the EPSPs. EPSP amplitudes were therefore measured by fitting the rising phase of each response with a model trace (red), as described in Materials and Methods.

resulting phenotype of these mice is therefore likely to be elevated levels of NT3, either in skeletal muscle itself or elsewhere.

\section{Monosynaptic input from muscle spindle afferents to MNs is} increased in mlc/NT3 mice

The clasping phenotype of the mlc/NT3 mice suggested a functional deficit in their motor pathways. To test this idea, we measured the monosynaptic inputs from Ia afferents innervating the quadriceps $(\mathrm{Q})$ and adductor (Add) muscles to MNs in spinal segments L3, L4, and L5. The input to all MNs in each segment was measured by recording the summed responses to each muscle nerve via a suction electrode on the ventral root (see Materials and Methods). Representative traces are shown in Figure 1. Normally, Q and Add Ia afferents provide relatively little input to MNs in the L5 segment, but in mlc/NT3 mice, this input was more than doubled (wild type: $15.6 \pm 1.9 \mu \mathrm{V}, n=10$; mlc/NT3: $38.1 \pm 6.9 \mu \mathrm{V}, n=12 ; p<0.01)$. At L4, there was also a large increase in the responses to $\mathrm{Q}$ and Add stimulation (wild type: $26.2 \pm 4.4, n=9$; mlc/NT3: $70.7 \pm 8.6, n=16$; $p<0.002)$. The responses recorded from the $\mathrm{L} 3$ ventral root were increased in mlc/NT3 mice by a smaller amount, but measurements of the Q and Add inputs were less reliable at this segmental level because the synaptic inputs to many MNs were suprathreshold even in wild-type mice (Fig. 1), complicating the measurement of the underlying synaptic potentials (wild type: $104.8 \pm 4.9, n=13$; mlc/NT3: $139.3 \pm 12.2 \mu \mathrm{V}, n=13 ; p<0.02)$. These results indicate that muscle afferents in mlc/NT3 mice provide stronger inputs to many MNs than in wild-type mice.

\section{Normal central projections of Ia sensory fibers in mlc/NT3 mice}

The stronger Ia inputs in mlc/NT3 mice suggested there were increased projections from Ia sensory neurons to the spinal cord. These changes might be visible as increased numbers or densities of Ia collaterals in the ventral horn. Prenatal exposure to elevated NT3 is known to result in increased numbers of trkC + sensory neurons, which include Ia and Ib fibers (Oakley et al., 1995; Ring-
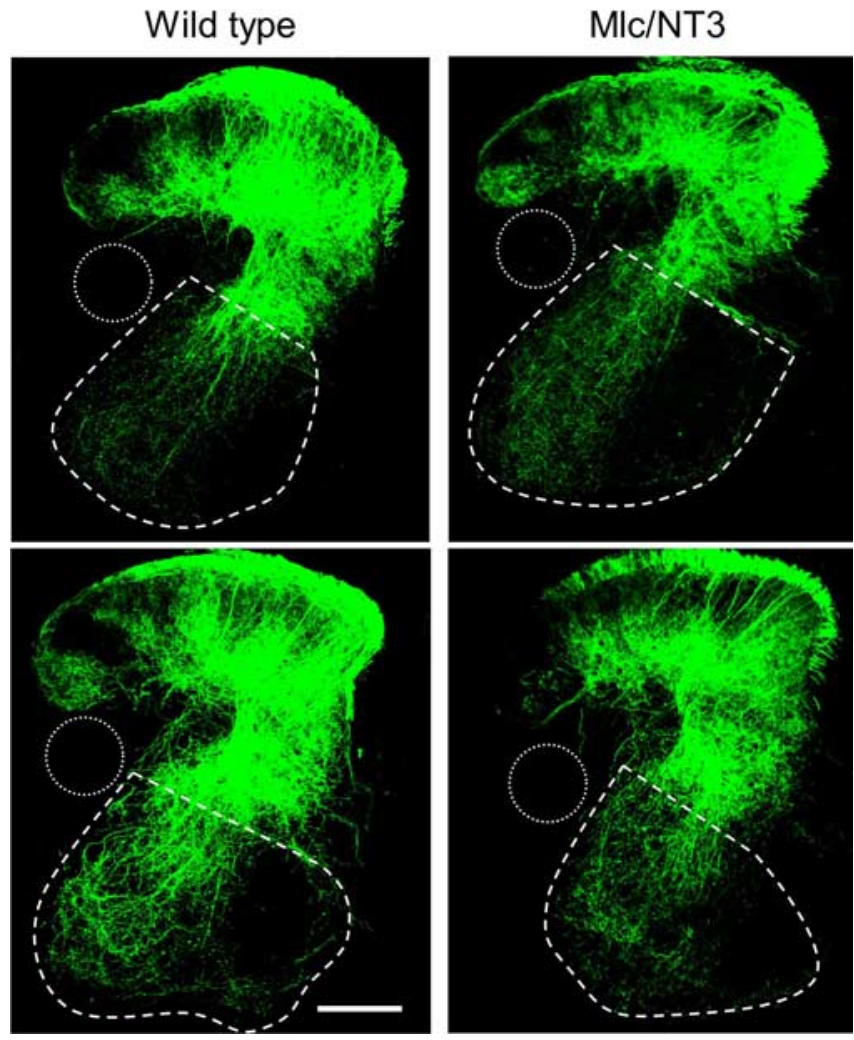

Figure 2. Central projections of sensory afferents in wild-type (left) and mlc/NT3 (right) mice at P7. Two examples for each genotype are provided to show the variability in density of la projections. The ROI used to measure the density of la projections is shown enclosed by a white dashed line. The ROl used to measure the background intensity is shown as a white dotted circle. Scale bar, $150 \mu \mathrm{m}$.

stedt et al., 1997). In the mlc/NT3 mice used in the present study, Taylor and co-workers (2001) reported a moderate increase in the number of large ( $>8 \mu \mathrm{m}$ diameter) sensory axons in the L5 dorsal root, as well as an increase in the relative number of large sensory neurons in the L5 DRG. These results suggest an increase in the number of Ia sensory neurons in mlc/NT3 mice. Moreover, elevated levels of NT3 can lead to exuberant growth and branching of large $\left(\operatorname{trkC}^{+}\right)$sensory neurons in vitro (Lentz et al., 1999; Ozdinler et al., 2004). Elevated NT3 levels in mlc/NT3 mice might therefore induce excess growth of Ia axons that provided the observed increase in Ia proprioceptive input.

We therefore assessed the central projections of sensory axons in the spinal cord for anatomical evidence of increased Ia projections into the ventral horn, where synaptic connections between Ia afferents and MNs are located. The cut proximal end of the L3 dorsal root was backfilled with fluorescent dextran to label the projections of Ia afferent collaterals in the ventral horn (Fig. 2). We made a semiquantitative assessment of Ia projections by measuring the percentage of pixels in this region with intensity above background (see Materials and Methods). Surprisingly, the Ia central projection into the ventral horn of spinal cord was no greater in mlc/NT3 mice than in wild-type mice (wild type: $18 \pm$ $2 \%, n=7 ;$ mlc/NT3: $20 \pm 1 \%, n=4 ; p=0.25)$. Although these techniques are unlikely to detect small changes in the density of Ia projections, these anatomical results suggest that the large increase in functional connectivity does not result from a major increase in anatomical projections. 


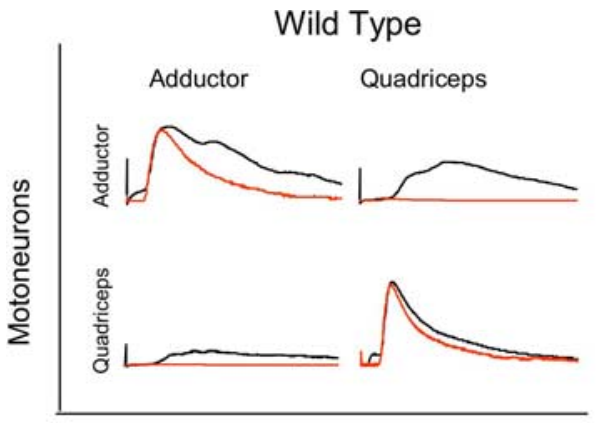

Sensory Afferents

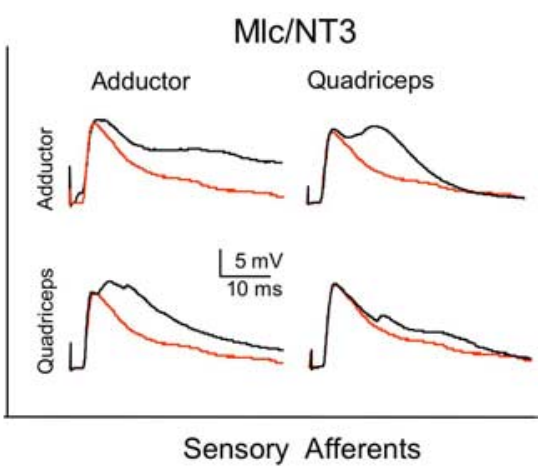

Figure 3. The specificity of monosynaptic connections between $Q$ and Add la afferents and MNs is disrupted in mlc/NT3 mice. Representative EPSPs (black traces) in $Q$ and Add MNs were elicited by stimulation of the $Q$ and Add muscle nerves in P7 mice. Model traces (red) were used to measure the monosynaptic component of each response. Note the presence of large monosynaptic components in unrelated $(Q \rightarrow$ Add and Add $\rightarrow Q$ ) EPSPs in mlc/NT3 mice. These EPSPs are absent in wild-type mice.

\section{Disruption of synaptic selectivity between Ia afferents and MNs in mlc/NT3 mice}

The behavioral phenotype and increased short-latency EPSPs in MNs of mlc/NT3 mice suggested either a global increase in synaptic connectivity between Ia afferents and MNs or a disruption in the pattern of these connections. To distinguish between these alternatives, we measured the input from muscle sensory neurons to identified MNs using intracellular recordings. Normally, Ia axons innervating muscle spindles in one muscle make strong, monosynaptic connections to MNs supplying the same muscle (homonymous connections) but much weaker connections to MNs supplying antagonist or functionally unrelated muscles (hereafter referred to as unrelated connections). We focused on MNs in the L3 and L5 spinal segments, for which detailed information is available concerning the specificity of synaptic connections between muscle sensory and motor neurons. At the L3 level, the $\mathrm{Q}$ and Add muscle groups provide a convenient test for specific connections between Ia sensory axons and MNs. The central projections of Ia afferents and the MNs for both muscle groups are located in the same spinal segments (McHanwell and Biscoe, 1981; Rivero-Melian, 1996). Despite the similarity in location of these MN pools, homonymous connections within each group are much stronger than unrelated ones between the two groups (Mears and Frank, 1997). At the L5 level, we chose the APFs as one group and the FDL muscle as the other. Homonymous connections within the APF group are strong, whereas inputs from FDL to any of the APF group are quite weak (Eccles et al., 1960).

Intracellular recordings revealed that the normal pattern of these connections was disrupted in mlc/NT3 mice (Fig. 3). In both wild-type and mlc/NT3 mice, homonymous connections within each of these four groups were strong. The homonymous inputs were $\sim 10 \mathrm{mV}$ for each group (Fig. 4), and the small differences between these inputs in wild-type versus mlc/NT3 mice were not significant ( $p=0.7$ for the $\mathrm{Q}-$ Add group and $p=0.5$ for the FDL-APF group). Unrelated inputs for wild-type and mlc/NT3 mice, however, were strikingly different. In wild-type mice, unrelated inputs were usually $<1 \mathrm{mV}$ but were $10-20$ times larger in mlc/NT3 mice (Fig. 4). These large differences were highly significant ( $p<0.001$ for both groups). Prenatal exposure to elevated levels of NT3 therefore leads to a loss of synaptic selectivity between Ia sensory axons and their unrelated MNs.

\footnotetext{
Aberrant connections in mlc/NT3 mice are monosynaptic

In addition to their monosynaptic inputs to homonymous MNs, muscle sensory axons also make polysynaptic connections to
}

other groups of MNs. For example, Ia axons evoke disynaptic inhibition in certain classes of MNs supplying antagonist muscles. The aberrant unrelated connections in mlc/NT3 mice might therefore be mediated polysynaptically. To obtain more direct evidence on this point, we performed several additional tests.

The simplest test was to measure the synaptic latencies of homonymous and unrelated inputs. The latencies of unrelated inputs in mlc/NT3 mice were within the range of homonymous input latencies in both strains [unrelated mlc/NT3, $3.6 \pm$ $0.1 \mathrm{~ms}(n=14)$; homonymous mlc/NT3, $3.5 \pm 0.04 \mathrm{~ms}(n=14)$; homonymous wild type, $4.0 \pm 0.03 \mathrm{~ms}(n=13)]$. In a separate series of experiments in wild-type mice of the same age, we found that the latency of the well characterized disynaptic projection from Q Ia afferents to posterior biceps-semitendinosis MNs (Eccles and Lundberg, 1958) is $\sim 2$ ms longer than the latency of monosynaptic connections ( $\mathrm{Z}$. Wang and E. Frank, unpublished observations). On the basis of latency, the unrelated inputs in mlc/NT3 mice are therefore mediated monosynaptically.

Additional evidence came from an analysis of the fluctuations in latencies of individual responses. Within a series of individual monosynaptic responses, the latencies vary only slightly, because conduction times and synaptic delays are nearly the same from trial to trial. For polysynaptic connections, however, fluctuations in the amplitudes of the synaptic potentials in the interneurons produce a variability in the time at which an action potential, and hence synaptic release, occurs. We measured the latencies of $>30$ single responses for each of several EPSPs (Fig. 5) as described in Materials and Methods. The SD of latencies for individual homonymous potentials was small in both mlc/NT3 and wild-type mice $(0.07 \pm 0.01$ vs $0.08 \pm 0.01 \mathrm{~ms}$, respectively; $n=7$ for each group), whereas for the longer-latency unrelated inputs in wildtype mice, the SD was $>10$ times larger $(1.11 \pm 0.46 \mathrm{~ms} ; n=7$; $p<0.05)$. Fluctuations in latency for unrelated inputs in $\mathrm{mlc} /$ NT3 mice, however, were as small as the homonymous ones $(0.07 \pm 0.02 \mathrm{~ms} ; n=7)$, providing strong evidence that these connections are mediated monosynaptically.

Finally, the latency of monosynaptic inputs should be constant during repetitive stimulation, although responses become smaller from synaptic fatigue. The latency of polysynaptic inputs, however, should increase as the synaptic potentials in the interneurons become smaller, because initiation of the action potential, and hence synaptic release, will be delayed. This prediction was also confirmed for these connections. Increasing the stimulus frequency from $0.3 \mathrm{~Hz}$ to $3 \mathrm{~Hz}$, causing a reduction in $\sim 50 \%$ in the amplitude of all responses, did not change significantly the latency of homonymous or unrelated connections in mlc/NT3 mice (latency increase, $0.08 \pm 0.03 \mathrm{~ms}$ and $0.10 \pm 0.04 \mathrm{~ms} ; n=4$ for each group of connections). In contrast, the latency of the unrelated (but not homonymous) connections was increased in wild-type mice (latency increase, $0.37 \pm 0.11 \mathrm{~ms}$ for unrelated connections vs $0.09 \pm 0.03 \mathrm{~ms}$ for homonymous connections; $n=5$ for both groups; $p<0.01$ ). There is, therefore, little doubt that these abnormal unrelated inputs in mlc/NT3 mice are mediated monosynaptically. 


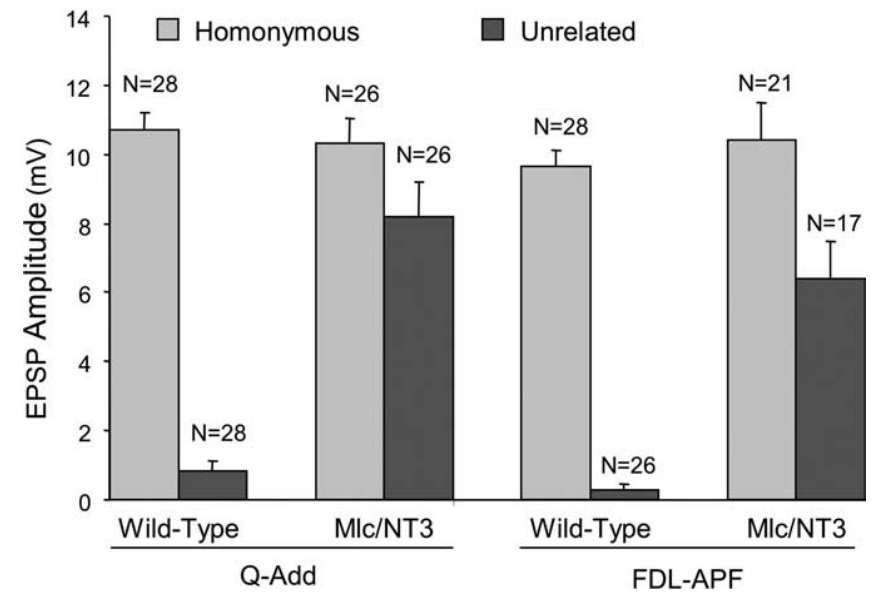

Figure 4. Average amplitudes ( $\pm 1 \mathrm{SE}$ ) of monosynaptic EPSPs recorded in MNs after stimulation of their homonymous and unrelated muscle inputs in P7 mice. In both the Q-Add and FDL-APF groups, homonymous EPSPs are large in both wild-type and mlc/NT3 mice and are not significantly different from each other. Only mlc/NT3 mice have large unrelated EPSPs, however, and the amplitudes of these EPSPs is significantly larger than those in wild-type mice $(p<0.001)$.

\section{Loss of synaptic selectivity occurs in individual mice}

The results presented so far do not distinguish between a major disruption of connections in only a subset of mlc/NT3 mice or a disruption in each individual mouse. To demonstrate that the loss occurs in each mlc/NT3 mouse, we devised a measure of synaptic specificity that provides an assessment of the selectivity of Ia afferents for homonymous versus unrelated MNs in individual mice (Sah and Frank, 1984). A specificity index (SI) was defined to compare the amplitudes of homonymous and unrelated inputs:

$$
\mathrm{SI} \equiv \frac{\mathrm{EPSP}_{\text {homo }}-\mathrm{EPSP}_{\text {unrelated }}}{\mathrm{EPSP}_{\text {homo }}+\mathrm{EPSP}_{\text {unrelated }}},
$$

where $\mathrm{EPSP}_{\text {homo }}$ is the average amplitude of monosynaptic inputs to homonymous MNs and $\mathrm{EPSP}_{\text {unrelated }}$ is the average amplitude of monosynaptic inputs to unrelated MNs.

This index is 1 when there are only homonymous inputs but falls to 0 when homonymous and unrelated inputs are of equal amplitude. As seen in Figure 6, the SIs for normal mice are high (average value, $0.92 \pm 0.02 ; n=18$ ), corresponding to homonymous inputs that are, on average, 24 times as large as unrelated ones. In contrast, the SIs for mlc/NT3 mice are much lower (average value, $0.23 \pm 0.05 ; n=22 ; p<0.001$ ), corresponding to homonymous inputs that are only 1.6 times larger than unrelated ones. All but one of the SI values for mlc/NT3 mice were smaller than any SI for wild-type mice, implying that the loss in synaptic selectivity occurs in every animal that was tested.

\section{Postnatal NT3 injections do not disrupt selectivity of Ia-MN connections}

To explore when increased levels of NT3 must be present to abrogate the selectivity of Ia-MN connectivity, we provided exogenous NT3 postnatally for 1 week in normal mice via local injections into the hindlimb. The effectiveness of these injections in raising levels of NT3 was confirmed by making intracellular recordings from MNs in Egr3 null mutant mice. These mice have weak Ia-MN synapses resulting from an absence of NT3 in postnatal muscle spindles, but functional transmission can be restored by postnatal NT3 injections (Chen et al., 2002). In the present experiments, these injections increased the homonymous inputs to Q and Add MNs in Egr3 null mutant mice from 0.08 to $11.45 \mathrm{mV}$, which is approximately the normal level (data not shown). Administration of NT3 (Adm/NT3) by injection produced only a small, and statistically insignificant, increase in homonymous Q and Add inputs (wild type: $10.7 \pm 0.5 \mathrm{mV}, n=$ 28; adm/NT3: $11.9 \pm 0.5 \mathrm{mV}, n=49)$, and unrelated inputs were virtually unchanged (wild type: $0.65 \pm 0.27 \mathrm{mV}, n=28$; Adm/ NT3: $0.69 \pm 0.23 \mathrm{mV}, n=49)$. A high level of selectivity (SI, $0.91 \pm 0.04$ ) was seen in all nine mice that were tested (Fig. 6, Adm/NT3). These results show that the disruption of synaptic selectivity is caused only by prenatal exposure to elevated levels of NT3, when the synapses are forming.

\section{Peripheral projections of sensory and motor axons are normal in mlc/NT3 mice}

A possible explanation for the disrupted synaptic selectivity is that individual Ia sensory axons might project to multiple muscles. If Ia axons normally supplying the Q muscle branched peripherally and supplied Add muscles as well, then stimulation of either the Q or the Add muscle nerve would evoke synaptic potentials in Q MNs even if the central projections were correct. We tested this possibility physiologically in four mlc/NT3 mice by determining whether any axons projected in both the $\mathrm{Q}$ and the Add nerves (Fig. 7). Stimulation of all the sensory axons in the L3 dorsal root evoked large, short-latency compound action potentials in both the Q and the Add nerves, confirming the presence of many group I afferents in both nerves. If any sensory or motor axons projected to both muscles, then stimulation of one muscle nerve should evoke action potentials in the other. No such potentials were recorded, however. In separate experiments, we found that single Ia afferents elicit an action potential of $\sim 10 \mu \mathrm{V}$ when recorded in this manner (data not shown) (see also Arber et al., 2000), so we would have detected the misprojection of a single sensory or motor axon. These results indicate that the aberrant synaptic connections do not result from abnormal peripheral projections of MN or Ia axons but instead from changes in their central connectivity within the spinal cord.

\section{Homonymous connections in mlc/NT3 mice are not saturated}

A possible explanation for this change in central connectivity is that prenatal exposure to increased NT3 levels causes a global increase in the strength of all Ia-MN connections. Postnatal injections of NT3 into Egr3 null mutant mice, for example, rescue the large reduction in these connections caused by the absence of NT3 in postnatal spindles in these mice (Chen et al., 2002). Although we did not observe an increase in homonymous EPSP amplitudes in mlc/NT3 mice (Fig. 4), it is possible that these homonymous projections had reached some upper limit of connectivity. In that case, a global increase in strength of Ia-MN connections would result in increased unrelated inputs without visible changes in homonymous ones. This could occur independently of any direct action of NT3 on the normal mechanisms responsible for determining synaptic selectivity. To examine this possibility, we sought additional evidence for any indication that homonymous inputs were already maximal in wild-type mice.

If normal homonymous connectivity was already at an upper limit, then homonymous EPSP amplitudes in mlc/NT3 mice would be even more tightly clustered at this limiting value. The expectation would be a reduction in the number of smallamplitude homonymous EPSPs. Rather than fewer small homonymous EPSPs, however, we found an increase in the number of 


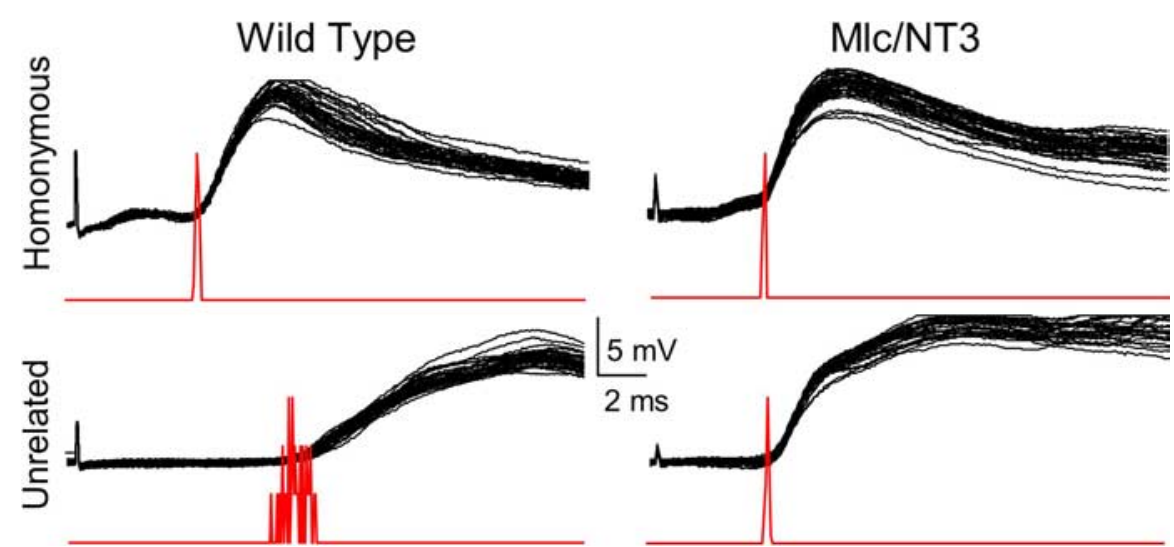

Figure 5. Fluctuations in synaptic latencies of homonymous and unrelated EPSPs in wild-type and $\mathrm{ml} / \mathrm{NT} 33$ mice. Each panel shows $>30$ superimposed single traces (black) of the same EPSP. The latency of each single trace was measured separately (see Materials and Methods), and a histogram of the individual latencies is shown in red below the traces. Unrelated EPSPs in wild-type mice have a longer and more variable latency than homonymous EPSPS. The latencies and latency fluctuations of unrelated EPSPS in mlc/NT3 mice, however, are the same as for homonymous EPSPs, providing strong evidence that they are mediated monosynaptically.

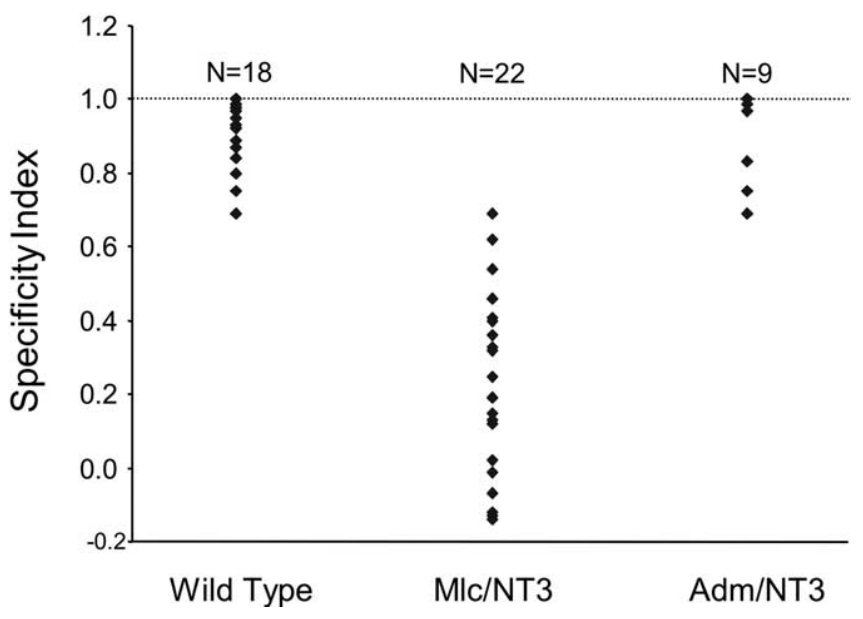

Figure 6. Specificity of la-MN EPSPs in individual mice, as measured by the SI (see Results for details) in individual mice. Synaptic specificity is high in both wild-type and adm/NT3 mice, but Sls are significantly lower in mlc/NT3 mice.

these small potentials in mlc/NT3 mice. This can be seen either in a conventional histogram of EPSP amplitude (Fig. $8 \mathrm{~A}$ ) or, perhaps more clearly, in a cumulative amplitude histogram (Fig. $8 B$ ). A convenient measure of this change in distribution can be obtained by defining small EPSPs as those with amplitudes $<7.0$ $\mathrm{mV}, \sim 1$ SD lower than the mean of homonymous EPSPs in wildtype mice. According to this definition, 9\% of the EPSPs in wild-type mice were small, whereas $\sim 28 \%$ were in this category in mlc/NT3 mice. Statistical tests reveal that these differences are significant ( $p<0.01$; see Materials and Methods). Small homonymous EPSPs were recorded in 8 of 15 mlc/NT3 mice and 4 of 14 wild-type mice, so the result is not attributable to the inclusion of a few mlc/NT3 mice with abnormally small EPSPs. The presence of substantial numbers of small-amplitude EPSPs in mlc/ NT3 mice argues against a global increase in Ia-MN synaptic connectivity. By elimination, the observation suggests instead that excess NT3 is disrupting some aspect of the normal mechanisms responsible for establishing specific Ia-MN connections.
Increased NT3 levels result in variability in motoneuronal receptivity to Ia inputs The wide variability in homonymous and unrelated EPSP amplitudes in mlc/NT3 mice, as seen in Figure 8, implies that some MNs receive less homonymous input than normal and some receive stronger unrelated inputs than their normal homonymous ones. This observation suggested that individual MNs might differ in their ability to attract a variety of Ia inputs, whether homonymous or unrelated. We tested this idea by comparing the strength of homonymous and unrelated inputs to individual MNs in mlc/NT3 mice. In wildtype mice, these inputs are not correlated with each other; the occasional large unrelated EPSP is not found preferentially in MNs with large homonymous inputs (Fig. 9A). In mlc/NT3 mice, however, homonymous and unrelated inputs to individual MNs are positively correlated (Fig. 9B). The amplitude of the unrelated input to an MN is generally similar to the amplitude of the homonymous input. This correlation is unlikely to result from the quality of the intracellular recordings, because EPSP amplitudes were not correlated with the resting potentials (Fig. 9C). The results suggest that some MNs are promiscuous, accepting substantial inputs from either homonymous (correct) or unrelated (incorrect) Ia axons, whereas others receive relatively little input from either source. Thus, in addition to a disruptive effect of elevated NT3 levels on synaptic selectivity in the Ia-MN system, extra NT3 also results in a variability among MNs to accept correct or incorrect Ia input.

\section{Discussion}

The neurotrophin NT3 plays a critical role in several aspects of the prenatal formation and postnatal function of the myotatic stretch reflex. During prenatal development, NT3 from peripheral sources is required for the survival of Ia afferents (Hory-Lee et al., 1993; Klein et al., 1994; Oakley et al., 1995; Wright et al., 1997). Prenatal NT3 is also required for the normal expression of the ETS gene ER81, which is expressed in all proprioceptive neurons and regulates the growth of Ia afferent collaterals into the ventral horn (Arber et al., 2000; Patel et al., 2003; Genc et al., 2004). Postnatally, NT3 expressed by muscle spindles maintains synaptic function between Ia afferents and MNs. If a motor nerve is cut in adult cats or rats, Ia-MN connections are reduced (Goldring et al., 1980) but can be restored and even made larger than normal by applying NT3 to the cut proximal end of the nerve (Munson et al., 1997; Mendell et al., 2001). NT3 injections also restore the loss of Ia-MN connectivity in Egr3 null mutant mice, in which NT3 expression by intrafusal fibers is eliminated (Chen et al., 2002). The NT3 released by muscle spindles may therefore modulate the strength of synaptic transmission between Ia afferents and MNs throughout life.

Given the multiple roles of NT3 in the development and maintenance of stretch reflexes, this neurotrophin might also be critical for the formation of specific patterns of Ia-MN connectivity. The embryonic overexpression of NT3 in skeletal muscle in mlc/NT3 transgenic mice provided an opportunity to assess the effects of excess NT3 levels on the development of the stretch reflex circuit. NT3 levels in skeletal muscles in these mice are increased severalfold (Taylor et al., 2001; present study), and 

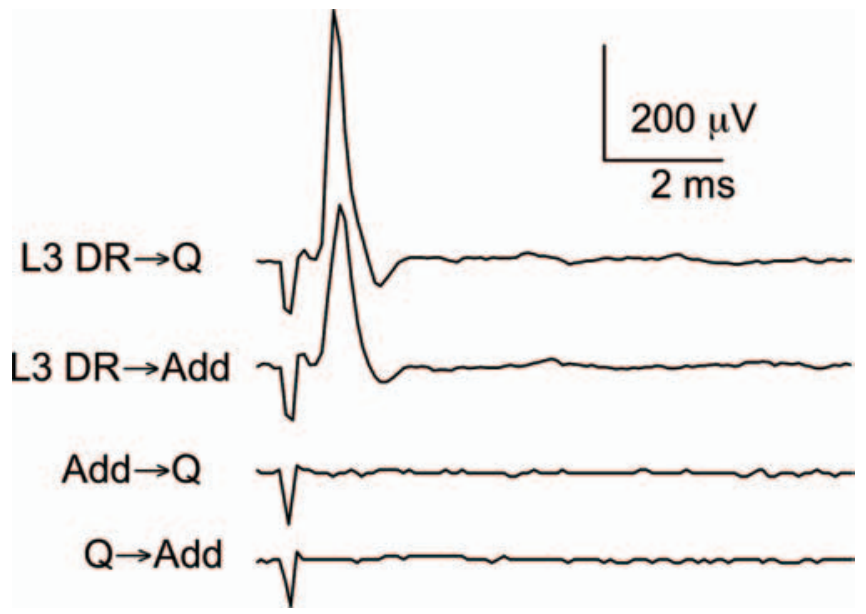

Figure 7. Peripheral axons of sensory and motor neurons do not project to multiple muscles in mlc/NT3 mice (P7). Stimulation of dorsal root L3 evoked compound action potentials in both the $Q$ and Add nerves (top 2 traces), indicating that both nerves contained sensory axons. Stimulation of one muscle nerve, however, evoked no action potentials in the other nerve, indicating that no sensory (or motor) nerve projected in both nerves.
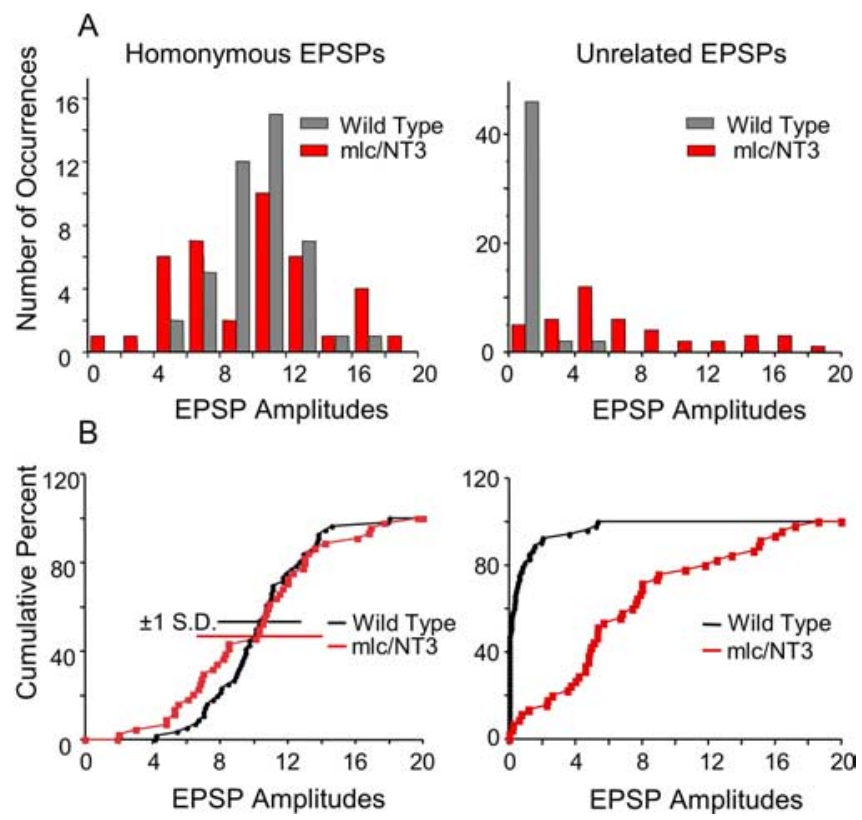

Figure 8. Amplitude histograms of monosynaptic EPSPs in wild-type and mlc/NT3 mice. Both conventional bar histograms $(\boldsymbol{A})$ and cumulative frequency histograms $(\boldsymbol{B})$ are presented for each data set. Amplitudes of homonymous EPSPs are more tightly clustered around the mean in wild-type (black bars and lines) compared with mlcNT3 (red bars and lines) mice. The SDs of EPSP amplitudes for each homonymous data set are shown as horizontal lines in $\boldsymbol{B}$ with the same color code used in the histograms. The SDs for homonymous EPSPs in wild-type and $\mathrm{mlc} / \mathrm{NT3}$ mice are significantly different from one another $(p<0.01$; see Materials and Methods).

their clenched digits, flexed hindlimbs, and abnormal gait suggested that stretch reflexes might be perturbed.

Extracellular recordings from ventral roots revealed that muscle afferents project more strongly to MNs in mlc/NT3 mice. A possible explanation was that an increase in the number of Ia sensory neurons was directly responsible for the larger Ia inputs to MNs. Embryonic overexpression of NT3 is known to result in increased numbers of muscle spindles and Ia afferents (Oakley et al., 1995; Wright et al., 1997; Li et al., 2006), including a moderate increase in the number of large-diameter sensory afferents in
mlc/NT3 mice (Taylor et al., 2001). We found no significant increase in the density of Ia collateral axons in the ventral horns of mlc/NT3 mice, however. Evidence from other studies has been mixed. Chicken embryos treated with exogenous NT3 do have increased central projections of Ia afferents into the ventral cord (Oakley et al., 1997). Overexpression of NT3 in the spinal cord via a transgene under the control of the nestin promoter, however, resulted in increased numbers of sensory neurons but a decrease in Ia projections to the ventral horn (Ringstedt et al., 1997). It is possible that the relatively late induction (E14) of NT3 in mlc/ NT3 mice (Taylor et al., 2001), after the onset of normally occurring cell death, is insufficient to produce increased Ia projections. In any case, the increased synaptic connectivity between Ia sensory neurons and MNs is unlikely to result simply from an increase in anatomical projections of Ia axon collaterals into the ventral horn.

Intracellular recordings from identified MNs revealed a striking change in the pattern of Ia-MN connectivity in mlc/NT3 mice. Although homonymous inputs were of normal amplitude, unrelated inputs were much larger than normal. These large, aberrant inputs provide an explanation for the large synaptic potentials recorded in the ventral roots, which measure the average Ia input to all motor axons projecting in the root. The projections to inappropriate MNs also could explain the abnormal posture and gait of these mice, because stretching one muscle would excite MNs projecting to many different muscles, resulting in inappropriate proprioceptive feedback.

Postnatal application of NT3 increases the amplitude of Ia-MN EPSPs, both in normal neonates and in mice lacking NT3 expression in muscle spindles (Seebach et al., 1999; Arvanov et al., 2000; Chen et al., 2002; Arvanian et al., 2003, 2006). NT3 expression in mlc/NT3 mice continues into adulthood (Taylor et al., 2001), so the aberrant synaptic inputs might result from postnatal exposure to elevated NT3. Postnatal injections of NT3 did not result in abnormal synaptic connectivity, however. In wildtype mice, the selective pattern of Ia-MN connections is already established at birth (Mears and Frank, 1997), so this result suggests that the disruption of synaptic selectivity by increased NT3 occurs during the formation of these synapses rather than subsequently.

A possible explanation for the loss of synaptic selectivity is that individual sensory and/or motor axons might project to multiple muscles, as seen after some peripheral nerve transection injuries (Aldskogius et al., 1987; Sumner, 1990). Electrophysiological tests demonstrated that single axons do not project to multiple muscles, however. The absence of peripheral projections of sensory or motor axons to multiple muscles argues that the defect in synaptic connectivity occurs centrally, within the spinal cord.

Elevated levels of NT3 might disrupt the selectivity of Ia-MN connections within the spinal cord by increasing the strength of these synapses globally. Chronic exposure to elevated levels of NT3 increase synaptic inputs to MNs in neonatal rats, and exogenous NT3 applied to the cut central end of muscle nerves potentiates synaptic inputs from Ia axons onto MNs above their normal amplitude even in adult rats and cats (Munson et al., 1997; Mendell et al., 2001; Arvanian et al., 2003). Exposure to elevated NT3 levels prenatally might therefore cause a similar increase in the strength of all Ia-MN synapses. Homonymous EPSPs are not potentiated in mlc/NT3 mice, however. The absence of potentiation is unlikely to result from saturation because these EPSPs can be potentiated in adult and neonatal rats by treatment with NT3 (Munson et al., 1997; Mendell et al., 2001; Arvanian et al., 2003). For the mice used in this study, the distribution of hom- 

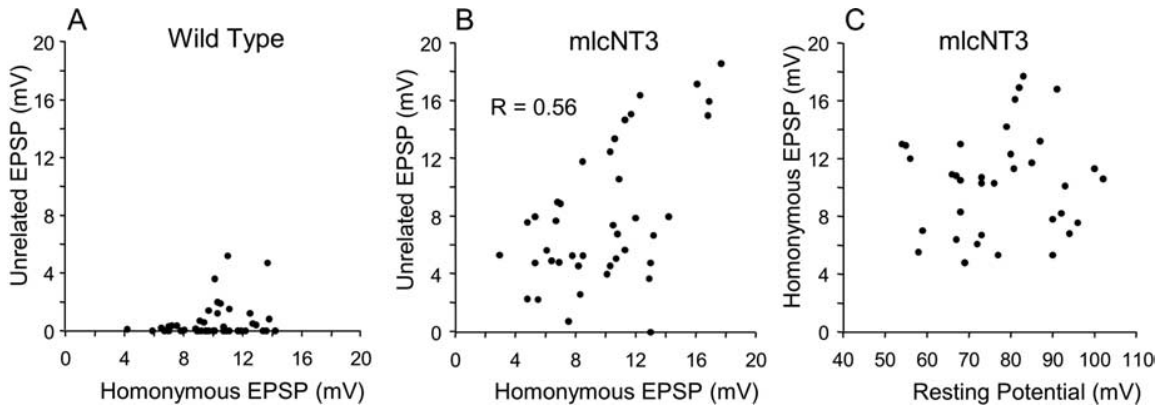

Figure 9. Homonymous and unrelated EPSP amplitudes are positively correlated in individual MNs in mlc/NT3 but not wildtype mice. Each point represents EPSP amplitudes $(\boldsymbol{A}-\boldsymbol{C})$ or resting potential $(\boldsymbol{C})$ in a single MN. The absence of a correlation between EPSP amplitude and resting potential $(\boldsymbol{C})$ suggests that the positive correlation between homonymous and unrelated EPSPS $(\boldsymbol{B})$ is not an artifact of the quality of the intracellular recording.

onymous EPSP amplitudes in mlc/NT3 mice also argues against a saturation of these potentials in normal mice. If homonymous EPSPs were already of maximal strength in wild-type mice, then their amplitudes would be even more tightly clustered around this maximum in mlc/NT3 mice, because the smaller homonymous EPSPs, not yet completely saturated, would be increased toward the maximum. Instead of a more tightly clustered distribution, however, homonymous EPSP amplitudes in these mice were more variable than normal, with significantly greater numbers of smaller EPSPs than in wild-type mice. Based on the previous experiments in adult rats and on the distribution of EPSP amplitudes in the mice used in this study, it is therefore unlikely that homonymous synaptic connections are already maximal in wild-type mice. Instead, these results imply that elevated levels of NT3 during embryonic development disrupt the mechanisms responsible for the formation of the selective pattern of synaptic connections between homonymous sensory and motor neurons.

Further analysis of EPSP amplitudes in individual MNs revealed an additional factor that contributes to the disrupted pattern of Ia-MN connections. The wide distribution in strengths of homonymous Ia inputs to individual MNs implies that the same population of Ia axons is massively innervating some MNs and mostly avoiding others. This marked variability in Ia input to different MNs is unlikely to occur simply by chance. Each muscle nerve we used contains at least 30-50 Ia axons (Kucera et al., 2002), which would tend to equalize synaptic input to each homonymous MN even if each Ia axon were making connections of random strength. Individual MNs within a single motor pool apparently have different receptivities to being innervated by their homonymous Ia axons. Some MNs receive even more homonymous input than in wild-type mice, whereas others are essentially refractory to these inputs.

The wide variability of homonymous EPSP amplitudes applies to unrelated inputs as well. We found that homonymous and unrelated EPSP amplitudes are positively correlated in individual MNs, suggesting that excess NT3 leads not only to a breakdown of synaptic specificity but also to a high degree of variability in the innervatibility of individual MNs.

What are the molecular mechanisms underlying the disruption of these connections by NT3? The formation of specific patterns of synaptic connections is likely to require the expression of recognition molecules such as cadherins or protocadherins (Obst-Pernberg and Redies, 1999; Shapiro and Colman, 1999; Takai et al., 2003; Takeichi, 2007) in both presynaptic and postsynaptic neurons. TrkC, the specific receptor for NT3, is expressed in both Ia sensory neurons and MNs (Schecterson and
Bothwell, 1992; McMahon et al., 1994; Wright and Snider, 1995; Copray and Kernell, 2000; Stephens et al., 2005), and NT3 is transported retrogradely back to their cell bodies (Buck et al., 2000). Elevated NT3 levels in muscle could therefore misregulate the expression of these recognition proteins in both cell classes. If recognition proteins were overexpressed or underexpressed in mlc/NT3 mice, then the pattern of Ia-MN connections would be disrupted. Specific cell adhesion molecules have been implicated in mediating synaptic specificity in the stretch reflex circuit (Price et al., 2002), so it will be interesting to determine whether the expression of any of these candidate proteins, in both sensory and motor neurons, is modified in mlc/NT3 mice.

The variability in innervatibility of MNs by both homonymous and unrelated Ia axons requires a variability in the MNs themselves. One possibility is that MNs within a single motor pool have variable input resistances and capacitances; the same amount of synaptic input would evoke smaller EPSPs in larger MNs. In addition to variations in overall cell size, differences in the extent or complexity of dendritic arbors could influence the number of synaptic contacts an MN receives from both correct and incorrect Ia axons. Different MNs also express different levels of trkC (Copray and Kernell, 2000), resulting in variations in the amounts of NT3 transported back to the cell body. If NT3 levels modulate the expression of genes that regulate synaptic input, the total amount of Ia input to an individual MN could also be related to the level of its trkC expression. Distinguishing among these or other possible explanations will require experiments that relate the total innervation of individual MNs to their morphologies and proteomic signatures, currently a technical challenge.

\section{References}

Aldskogius H, Molander C, Persson J, Thomander L (1987) Specific and nonspecific regeneration of motor axons after sciatic nerve injury and repair in the rat. J Neurol Sci 80:249-257.

Arber S, Ladle DR, Lin JH, Frank E, Jessell TM (2000) ETS gene Er81 controls the formation of functional connections between group Ia sensory afferents and motor neurons. Cell 101:485-498.

Arvanian VL, Horner PJ, Gage FH, Mendell LM (2003) Chronic neurotrophin-3 strengthens synaptic connections to motoneurons in the neonatal rat. J Neurosci 23:8706-8712.

Arvanian VL, Bowers WJ, Anderson A, Horner PJ, Federoff HJ, Mendell LM (2006) Combined delivery of neurotrophin-3 and NMDA receptors 2D subunit strengthens synaptic transmission in contused and staggered double hemisected spinal cord of neonatal rat. Exp Neurol 197:347-352.

Arvanov VL, Seebach BS, Mendell LM (2000) NT-3 evokes an LTP-like facilitation of AMPA/kainate receptor-mediated synaptic transmission in the neonatal rat spinal cord. J Neurophysiol 84:752-758.

Buck CR, Seburn KL, Cope TC (2000) Neurotrophin expression by spinal motoneurons in adult and developing rats. J Comp Neurol 416:309-318.

Chen HH, Tourtellotte WG, Frank E (2002) Muscle spindle-derived neurotrophin 3 regulates synaptic connectivity between muscle sensory and motor neurons. J Neurosci 22:3512-3519.

Chen HH, Hippenmeyer S, Arber S, Frank E (2003) Development of the monosynaptic stretch reflex circuit. Curr Opin Neurobiol 13:96-102.

Coppola V, Kucera J, Palko ME, Martinez-De Velasco J, Lyons WE, Fritzsch B, Tessarollo L (2001) Dissection of NT3 functions in vivo by gene replacement strategy. Development 128:4315-4327.

Copray S, Kernell D (2000) Neurotrophins and trk-receptors in adult rat spinal motoneurons: differences related to cell size but not to "slow/fast" specialization. Neurosci Lett 289:217-220.

Eccles JC, Eccles RM, Lundberg A (1957) The convergence of monosynaptic 
excitatory afferents onto many different species of alpha motoneurones. J Physiol (Lond) 137:22-50.

Eccles JC, Eccles RM, Magni F (1960) Monosynaptic excitatory action on motoneurones regenerated to antagonistic muscles. J Physiol (Lond) 154:68-88.

Eccles RM, Lundberg A (1958) The synaptic linkage of direct inhibition. Acta Physiol Scand 43:204-215.

Ernfors P, Lee K-F, Kucera J, Jaenisch R (1994) Lack of neurotrophin-3 leads to deficiencies in the peripheral nervous system and loss of limb proprioceptive afferents. Cell 77:503-512.

Frank E, Westerfield M (1983) Development of sensory-motor synapses in the spinal cord of the frog. J Physiol (Lond) 343:593-610.

Genc B, Ozdinler PH, Mendoza AE, Erzurumlu RS (2004) A chemoattractant role for NT-3 in proprioceptive axon guidance. PLoS Biol 2:e403.

Goldring JM, Kuno M, Nunez R, Snider WD (1980) Reaction of synapses on motoneurones to section and restoration of peripheral sensory connexions in the cat. J Physiol (Lond) 309:185-198.

Hory-Lee F, Russell M, Lindsay RM, Frank E (1993) Neurotrophin 3 supports the survival of developing muscle sensory neurons in culture. Proc Natl Acad Sci USA 90:2613-2617.

Klein R (1994) Role of neurotrophins in mouse neuronal development. FASEB J 8:738-744.

Klein R, Silos-Santiago RJ, Smeyne RJ, Lira SA, Brambilla R, Bryant S, Zhang L, Snider WD, Barbacid M (1994) Disruption of the neurotrophin-3 receptor gene trkC eliminates Ia muscle afferents and results in abnormal movements. Nature 368:249-251.

Kucera J, Cooney W, Que A, Szeder V, Stancz-Szeder H, Walro J (2002) Formation of supernumerary muscle spindles at the expense of Golgi tendon organs in ER81-deficient mice. Dev Dyn 223:389-401.

Lentz SI, Knudson CM, Korsmeyer SJ, Snider WD (1999) Neurotrophins support the development of diverse sensory axon morphologies. J Neurosci 19:1038-1048.

Li LY, Wang Z, Sedy J, Quazi R, Walro JM, Frank E, Kucera J (2006) Neurotrophin-3 ameliorates sensory-motor deficits in Er81-deficient mice. Dev Dyn 235:3039-3050.

Lichtman JW, Jhaveri S, Frank E (1984) Anatomical basis of specific connections between sensory axons and motor neurons in the bullfrog's brachial spinal cord. J Neurosci 4:1754-1763.

Lindsay RM (1996) Role of neurotrophins and trk receptors in the development and maintenance of sensory neurons: an overview. Philos Trans R Soc Lond B Biol Sci 351:365-373.

McHanwell S, Biscoe TJ (1981) The localization of motoneurons supplying the hindlimb muscles of the mouse. Philos Trans R Soc Lond B Biol Sci 293:477-508.

McMahon SB, Armanini MP, Ling LH, Phillips HS (1994) Expression and coexpression of Trk receptors in subpopulations of adult primary sensory neurons projecting to identified peripheral targets. Neuron 12:1161-1171.

Mears SC, Frank E (1997) Formation of specific monosynaptic connections between muscle spindle afferents and motoneurons in the mouse. J Neurosci 17:3128-3135.

Mendell LM, Munson JB, Arvanian VL (2001) Neurotrophins and synaptic plasticity in the mammalian spinal cord. J Physiol (Lond) 533:91-97.

Mendelson B, Frank E (1991) Specific monosynaptic sensory-motor connections in the chick embryonic spinal cord form in the absence of patterned neural activity and motoneuronal cell death. J Neurosci 11:1390-1403.

Munson JB, Johnson RD, Mendell LM (1997) NT-3 increases amplitude of EPSPs produced by axotomized group Ia afferents. J Neurophysiol 77:2209-2212.

Oakley RA, Garner AS, Large TH, Frank E (1995) Muscle sensory neurons require neurotrophin-3 from peripheral tissues during the period of normal cell death. Development 121:1340-1350.
Oakley RA, Lefcort FB, Clary DO, Reichardt LF, Prevette D, Oppenheim RW, Frank E (1997) Neurotrophin-3 promotes the differentiation of muscle spindle afferents in the absence of peripheral targets. J Neurosci 17:4262-4274.

Obst-Pernberg K, Redies C (1999) Cadherins and synaptic specificity. J Neurosci Res 58:130-138.

Ozdinler PH, Ulupinar E, Erzurumlu RS (2004) Local neurotrophin effects on central trigeminal axon growth patterns. Dev Brain Res 151:55-66.

Patel TD, Kramer I, Kucera J, Niederkofler V, Jessell TM, Arber S, Snider WD (2003) Peripheral NT3 signaling is required for ETS protein expression and central patterning of proprioceptive sensory afferents. Neuron 38:403-416.

Price SR, De Marco Garcia NV, Ranscht B, Jessell TM (2002) Regulation of motor neuron pool sorting by differential expression of type II cadherins. Cell 109:205-216.

Ringstedt T, Kucera J, Lendahl U, Ernfors P, Ibanez CF (1997) Limb proprioceptive deficits without neuronal loss in transgenic mice overexpressing neurotrophin-3 in the developing nervous system. Development 124:2603-2613.

Rivero-Melian C (1996) Organization of hindlimb nerve projections to the rat spinal cord: a choleragenoid horseradish peroxidase study. J Comp Neurol 364:651-663.

Sah DW, Frank E (1984) Regeneration of sensory-motor synapses in the spinal cord of the bullfrog. J Neurosci 4:2784-2791.

Schecterson LC, Bothwell M (1992) Novel roles for neurotrophins are suggested by BDNF and NT-3 mRNA expression in developing neurons. Neuron 9:449-463.

Seebach BS, Arvanov V, Mendell LM (1999) Effects of BDNF and NT-3 on development of $\mathrm{Ia} /$ motoneuron functional connectivity in neonatal rats. J Neurophysiol 81:2398-2405.

Shapiro L, Colman DR (1999) The diversity of cadherins and implications for a synaptic adhesive code in the CNS. Neuron 23:427-430.

Sokal RR, Rohlf FJ (1995) Biometry: the principles and practice of statistics in biological research, Ed 3. New York: Freeman and Co.

Stephens HE, Belliveau AC, Gupta JS, Mirkovic S, Kablar B (2005) The role of neurotrophins in the maintenance of the spinal cord motor neurons and the dorsal root ganglia proprioceptive sensory neurons. Int J Dev Neurosci 23:613-620.

Sumner AJ (1990) Aberrant reinnervation. Muscle Nerve 13:801-803.

Takai Y, Shimizu K, Ohtsuka T (2003) The roles of cadherins and nectins in interneuronal synapse formation. Curr Opin Neurobiol 13:520-526.

Takeichi M (2007) The cadherin superfamily in neuronal connections and interactions. Nat Rev Neurosci 8:11-20.

Taylor MD, Vancura R, Patterson CL, Williams JM, Riekhof JT, Wright DE (2001) Postnatal regulation of limb proprioception by muscle-derived neurotrophin-3. J Comp Neurol 432:244-258.

Tessarollo L, Vogel KS, Palko ME, Reid SW, Parada LF (1994) Targeted mutation in the neurotrophin-3 gene results in loss of muscle sensory neurons. Proc Natl Acad Sci USA 91:11844-11848.

Ulupinar E, Jacquin MF, Erzurumlu RS (2000) Differential effects of NGF and NT-3 on embryonic trigeminal axon growth patterns. J Comp Neurol 425:202-218.

Wright DE, Snider WD (1995) Neurotrophin receptor mRNA expression defines distinct populations of neurons in rat dorsal root ganglia. J Comp Neurol 351:329-338.

Wright DE, Zhou L, Kucera J, Snider WD (1997) Introduction of a neurotrophin-3 transgene into muscle selectively rescues proprioceptive neurons in mice lacking endogenous neurotrophin- 3. Neuron 19:503-517.

Zhou L, Baumgartner BJ, Hill-Felberg SJ, McGowen LR, Shine HD (2003) Neurotrophin-3 expressed in situ induces axonal plasticity in the adult injured spinal cord. J Neurosci 23:1424-1431. 